\title{
Discotic Mesophases of the Hexakis(alkylsulfono)benzene Series: Characterization by Differential Scanning Calorimetry, Optical Microscopy and Nuclear Magnetic Resonance Spectroscopy [1]
}

\author{
K. Praefcke* and W. Poules \\ Institut für Organische Chemie, Technische Universität Berlin, \\ Straße des 17. Juni 135, D-1000 Berlin 12, Germany \\ B. Scheuble \\ E. Merck, Frankfurter Straße 250, D-6100 Darmstadt 1, Germany \\ R. Poupko and Z. Luz* \\ The Weizmann Institute of Science, 76100 Rehovot, Israel \\ Z. Naturforsch. 39b, 950-956 (1984); received February 2, 1984 \\ Hexakis(alkylsulfono)benzene Series, NMR Spectra \\ Members of the Hexakis(alkylsulfono)benzene series $\mathbf{4}$, containing 7 to 15 carbon atoms per \\ alkyl chain $(\mathbf{4} \mathbf{c}-\mathbf{k})$, exhibit one and possibly several liquid crystalline discotic mesophases. The \\ mesophases are uniaxial and most probably have a columnar structure. Their anisotropic magne- \\ tic susceptibility is negative and in sufficiently strong magnetic fields they align with the director \\ perpendicular to the field direction. Deuterium NMR spectra of $\mathrm{C}_{6} \mathrm{D}_{6}$ dissolved in the mesophases \\ exhibit quadrupolar splittings which are strongly temperature dependent. In analogy with the \\ conventional discotics this suggests the presence of multiple solvation sites for solute molecules.
}

\section{Introduction}

In recent years a new class of liquid crystalline compounds was discovered consisting of disc-like rather than rod-like molecules [2,3]. These compounds mostly consist of a central unsaturated core to which alkyl chains are bonded at the periphery. Typical examples of aromatic cores are benzene, triphenylene and truxene, but other cores of lower symmetry are also known [2]. Very recently also cyclohexane as the first alicyclic, saturated core of discotic liquid crystals has been reported [4]. The alkyl chains (usually six) are bonded to the central core by oxygen bridges using ether, ester or benzoate functional groups. Some months ago two of us (K. P. and W. P.) [5] have for the first time synthesized three members of a homologous series of discotic compounds $(\mathbf{4} \mathbf{e}, \mathbf{g}, \mathbf{i})$ in which the bridging link is a sulfur rather than an oxygen atom (see reaction scheme and experimental part). Specifically a sulfone bridging group was used and the series consisted of hexakis(alkylsulfono)benzenes (4).

In the present paper we describe a study of the phase diagram of this series using differential scanning calorimetry (DSC), optical microscopy (OM) and nuclear magnetic resonance spectroscopy (NMR). It will be shown that mesophases occur in

\footnotetext{
* Reprint requests to Prof. Dr. K. Praefcke or Z. Luz. 0340-5087/84/0700-0950/\$ 01.00/0
}

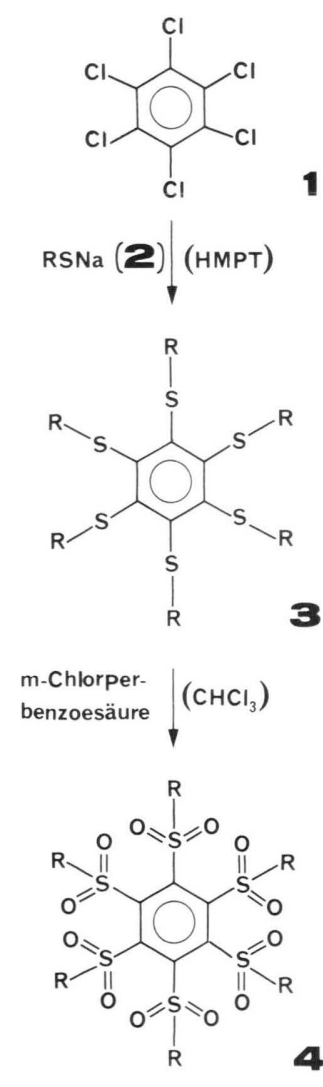

a-l: $R=n-C_{n} H_{2 n+1} \quad(n=5-16)$ 
members of the series 4 for which $n$, the number of carbon atoms per chain, ranges between 7 and 15 $(\mathbf{4} \mathbf{c}-\mathbf{k})$. The mesophases have apparently columnar structure with hexagonal symmetry. The are aligned by strong magnetic fields with the director perpendicular to the field direction (i.e. $\Delta \chi<0$ ).

\section{Experimental}

\section{A. Preparation of compounds}

The hexasulfones 4 were synthesized as described in ref. [5] by first reacting hexachlorobenzene (1) with the corresponding sodium alkylthiolates $(\mathbf{2} \mathbf{a}-\mathbf{l})$ in HMPT, to obtain the hexakis(alkylthio)benzenes $3 \mathbf{a}-\mathbf{l}$ (the yields ranged between 54 and $95 \%$ depending on the compound). These hexathioethers $\mathbf{3}$ with alkyl chains from $n$-pentyl to $n$-decyl $(3 \mathbf{a}-\mathbf{f})$ are yellow highly viscous oils at room temperature; those with alkyl chains from $n$-undecyl to $n$-hexadecyl $(\mathbf{3 g}-\mathbf{l})$ are yellowish crystalline materials, with melting points (by optical microscopy) of $29-30{ }^{\circ} \mathrm{C}$, $39,7-40,3{ }^{\circ} \mathrm{C}, 44-45{ }^{\circ} \mathrm{C}, 48-50{ }^{\circ} \mathrm{C}, 53,5-55^{\circ} \mathrm{C}$ and $57-59^{\circ} \mathrm{C}$, respectively. None of these compounds 3a-l show liquid crystalline properties [5]. Oxidation of the hexakis(alkylthio)benzenes $\mathbf{3}$ with $\mathrm{m}$ chloroperbenzoic acid gave the hexasulfones 4 (yields between 66 and $92 \%$ ) as nearly colourless crystalline substances. All compounds of type $\mathbf{3}$ and $\mathbf{4}$ were purified by column chromatography on aluminiumoxide/petrolether $\left(30-70{ }^{\circ} \mathrm{C}\right) /$ ether $(94: 6$ ratio) and recrystallisation from acetone/petrolether $\left(30-70{ }^{\circ} \mathrm{C}\right)$ or ethanol/petrolether $\left(30-70{ }^{\circ} \mathrm{C}\right)($ both $10: 1$ ratio). The melting and clearing points of the hexasulfones $(\mathbf{4} \mathbf{a}-\mathbf{l})$ obtained by DSC are given in Table I. The structures of all these new compounds of type 3 and $\mathbf{4}$ were confirmed by elementary analyses, mass spectroscopy, ${ }^{1} \mathrm{H}$, and ${ }^{13} \mathrm{C}$ NMR spectroscopy.

\section{B. DSC}

Two independent sets of differential scanning calorimetry measurements were performed using respectively a du Pont 1090, and a Mettler TA 3000 system. Usually 2 to $8 \mathrm{mg}$ of the compound were used and the temperature changed at a rate of $4-10 \% \mathrm{~min}$. Both increasing and decreasing temperature runs were recorded, and usually repeated several times on the same sample.

\section{Optical Microscopy}

Optical microscopy studies were performed using a Mettler FP 52 hot stage and temperature controller together with a standard Zeiss polarising micro- scope. Pictures of the viewing field were taken with transmission light using crossed polarized light.

\section{NMR Measurements}

NMR measurements were made of the deuteron signal of deuterated benzene dissolved (2.9 to $3.6 \mathrm{wt} . \%)$ in the mesophase. A Bruker WH 270 spectrometer operating at $41.45 \mathrm{MHz}$ for the deuteron resonance was used. The measurements were performed by first heating the samples outside the magnetic field to above the clearing point, and after vigorous shaking inserting it into the preheated NMR probe. The temperature was then slowly reduced from the isotropic phase into the mesophase and crystalline regions.

\section{Results and Discussion}

\section{A. $D S C$}

The phase transition temperatures and enthalpies of $\mathbf{4 a - l}(n=5-16)$ were studied using the differential scanning calorimetry (DSC) method. The lower members of the series $(\mathbf{4} \mathbf{a}, \mathbf{b}, n \leq 6)$ do not show a mesophase and they exhibit a high melting point which decreases monotonically with the alkyl chain

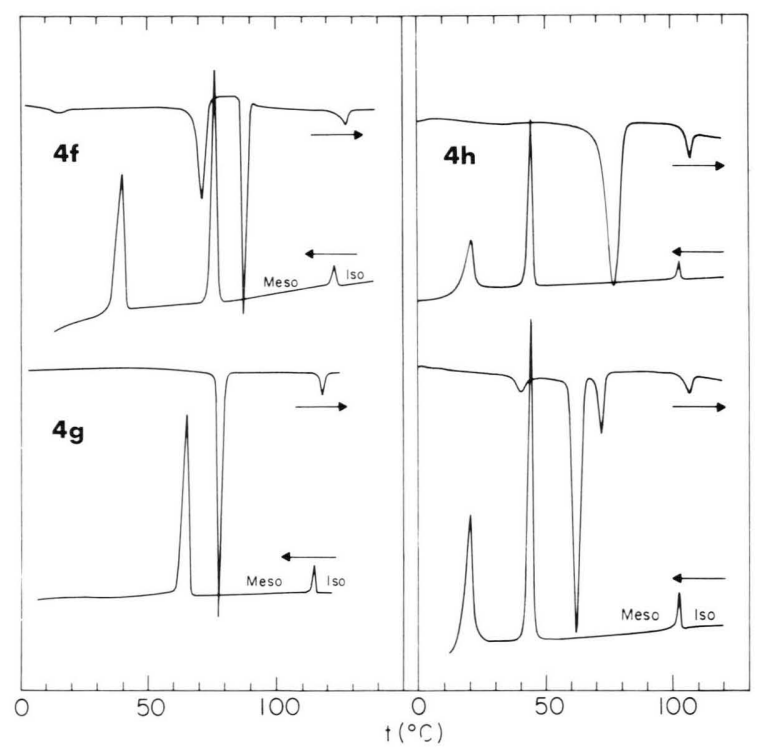

Fig. 1. Differential Scanning Calorimetry (DSC) traces for three homologues of the hexasulfone series $4 \mathbf{f}-\mathbf{h}$. The results were recorded with a scan rate of $10^{\circ} / \mathrm{min}$, for both increasing and decreasing the temperature. The results for $4 \mathrm{~h}$ include traces taken of a freshly prepared sample (upper part of the figure) and traces obtained from the same sample immediately after the first heating-cooling cycle (lower part). 


\begin{tabular}{lccccc}
\hline $\mathbf{4}$ & $n$ & Iso $\rightarrow$ Solid & Iso $\rightarrow$ Meso & Meso $\rightarrow$ Solid & Solid $\rightarrow$ Solid \\
\hline $\mathbf{a}$ & 5 & $226(16.8)$ & - & - & $55(7.1)$ \\
$\mathbf{b}$ & 6 & $164(18.8)$ & - & - & - \\
$\mathbf{c}$ & 7 & - & $138(3.7)$ & $120(30.0)$ & - \\
$\mathbf{d}$ & 8 & - & $134(4.8)$ & $108(33.3)$ & - \\
$\mathbf{e}$ & 9 & - & $130(4.7)$ & $87(37.9)$ & - \\
$\mathbf{f}$ & 10 & - & $125(4.1)$ & $77(46.5)$ & $40(45.9)$ \\
$\mathbf{g}$ & 11 & - & $115(5.5)$ & $65(56.0)$ & - \\
$\mathbf{h}$ & 12 & - & $103(4.1)$ & $45(54.1)$ & $20(34.6)$ \\
$\mathbf{i}$ & 13 & - & $90(3.8)$ & $44(66.5)$ & - \\
$\mathbf{j}$ & 14 & - & $77(2.6)$ & $42(67.1)$ & - \\
$\mathbf{k}$ & 15 & - & $63(2.4)$ & $49(75.8)$ & - \\
$\mathbf{l}$ & 16 & $69(109)$ & - & - & - \\
\hline
\end{tabular}

Table I. Transition temperatures (and enthalpies) of the observed transitions in the hexasulfone series 4 studied in the present work $^{\mathrm{a}}$.

\footnotetext{
a The temperatures are in ${ }^{\circ} \mathrm{C}$ and the enthalpies (which are given in brackets) are in $\mathrm{kJ} / \mathrm{mole}$.
}

length. $\mathbf{4} \mathbf{c}-\mathbf{k}$ possess (at least) on mesophase, but the DSC curves were not completely reproducible on cooling and heating of the samples. Some examples of DSC traces are shown in Fig. 1. For $\mathbf{4} \mathbf{g}$ two peaks corresponding respectively to the solid $\rightarrow$ mesophase and mesophase $\rightarrow$ liquid transitions are observed. It may be seen that there is considerable hysteresis in the low temperature transition. A similar effect may be seen in the results for $\mathbf{4} \mathbf{f}$, however for this compound there is in addition at least one more peak which apparently corresponds to another mesophase or to a solid $\rightarrow$ solid transition. In some cases the results for two successive heating runs were not reproducible. For example, on first heating $\mathbf{4 h}$ two DSC peaks were observed (corresponding respectively to melting and clearing points), while after cooling and reheating one and perhaps two additional peaks appeared in the DSC trace. On the other hand in all runs, cooling the sample gave reproducible traces.

A summary of the experimental transition temperatures and enthalpies is given in Table I and is plotted in the form of a phase diagram in Fig. 2. The values quoted in the table are from the cooling DSC traces since, as explained above, these results were found to be more reproducible, while in the figure transition temperatures for both heating and cooling cycles are given. It may be seen that for $\mathbf{4 c}$ to $\mathbf{4 k}$ cooling the isotropic liquid yields a liquid crystalline mesophase, while no such mesophases are found for $\mathbf{4 1}$ and higher homologues, or $\mathbf{4 b}$ and lower homologues. Thus there appears to be an "island of stability" for the mesophases of the hexasulfone series 4 ranging from $n=7$ to $15(\mathbf{4} \mathbf{c}-\mathbf{k})$. Note that not always does the mesophase transform on cooling to the thermodynamically stable solid phase: in some cases one or several intermediate phases occur below the mesophase region. It is not clear whether these intermediates are solid or perhaps also liquid crystalline mesophases. The NMR results reported below suggest that the latter may perhaps apply.

\section{B. Optical microscopy}

To further characterize the phases of the hexasulfone series 4 a phase diagram of this series of com-

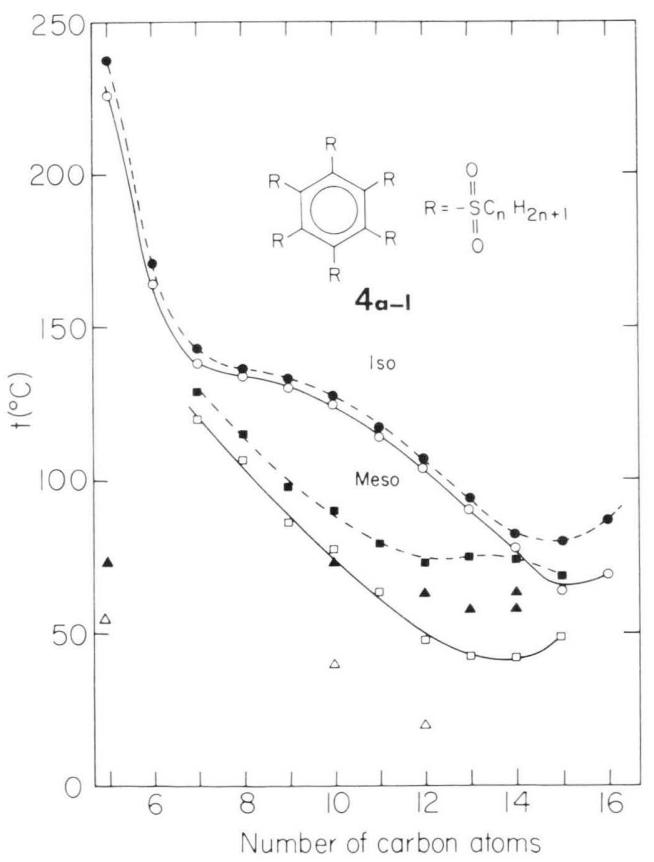

Fig. 2. Phase diagram of the hexasulfone series $\mathbf{4 a - 1}$ (for $5<n<16$ ) based on DSC measurements. The filled symbols correspond to transitions observed upon increasing of temperature while the open symbols to decreasing temperature. The circles correspond to transitions to or from the isotropic phase while squares to transitions to or from the normal mesophase. Triangles represent transitions between intermediate (solid) phases. 
pounds was constructed by optical microscopy. As may be seen from the results plotted in Fig. 3 it is almost identical to that obtained by the DSC method (Fig. 2). The texture of the mesophase observed in the $\mathbf{4} \mathbf{c}$ to $\mathbf{4 k}$ homologs just below the clearing point is essentially the same in all compounds and resemble the star shaped "flowers" shown in plates $137-140$ of ref. [6]. A typical picture corresponding to the mesophase of $\mathbf{4 f}$ is shown in Fig. 4.

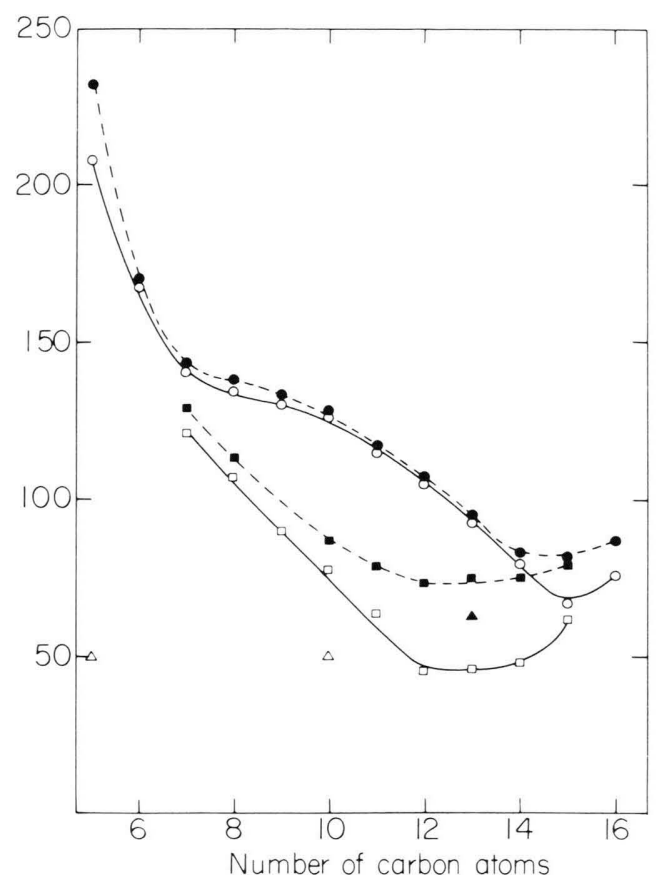

Fig. 3. Phase diagram for the hexasulfone series $4 \mathbf{a}-\mathbf{l}$ based on optical microscopic measurements. The symbols are as in Fig. 2.

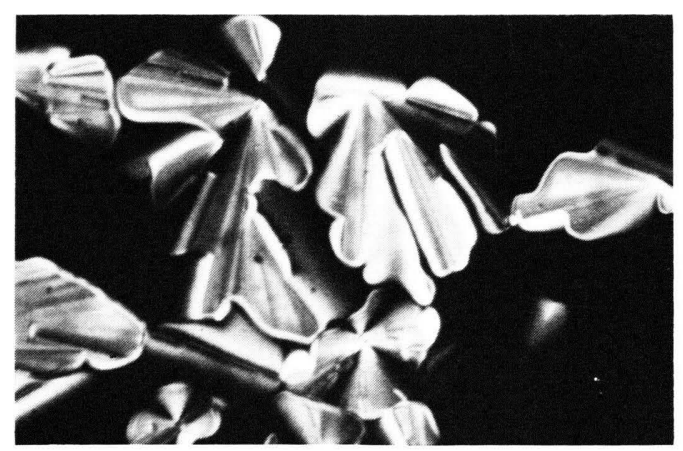

Fig. 4. A photographic picture of the texture of the mesophase of $\mathbf{4 f}$ taken at $124{ }^{\circ} \mathrm{C}$ using a polarizing microscope $($ Magnification $\times 160)$.
The solid phases of $\mathbf{4 a}$ and $\mathbf{4 b}$ below the liquid phase and of $\mathbf{4 c}$ to $\mathbf{4 h}$ below the mesophase exhibit a texture of long crystals which form a mosaic array. However different textures are observed for the intermediate phases of $\mathbf{4 i}$ and $\mathbf{4 j}$. Also the solids of $\mathbf{4 k}$ and $\mathbf{4 1}$ exhibit a powder-like texture rather than the crystalline mosaic shown by the lower homologs.

\section{NMR measurements}

NMR and particularly deuterium NMR, proved to be a most powerful tool for characterizing the orientational properties of liquid crystalline mesophases. The most direct way of employing this technique is via the NMR of deuterated mesogens. In the absence of such deuterated compounds we used an alternative approach in which the deuterium NMR spectrum of a deuterated solute (benzene- $\mathrm{d}_{6}$ ) introduced in small quantities $(2.9-3.6 \mathrm{wt} . \%)$ into the mesophase was recorded as function of various experimental conditions. In a liquid crystalline mesophase the deuterium spectrum exhibits quadrupole splittings which reflect the ordering and symmetry of the system [7]. The introduction of probe molecules slightly affects the phase transition temperatures but the gross features of the mesophases are not affected as long as the solute concentration is small.

A typical deuterium spectrum of a sample consisting of 2.9 wt. $\% \mathrm{C}_{6} \mathrm{D}_{6}$ in $\mathbf{4 h}$ and obtained by slowly cooling the isotropic solution into the mesophase region within the magnetic field is shown in the upper trace of Fig. 5 . The fact that a single doublet with

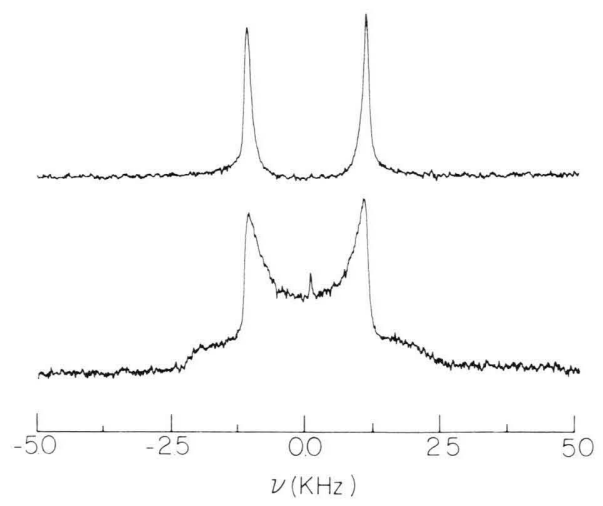

Fig. 5. Deuterium NMR spectra of a 2.9 wt. \% solution of $\mathrm{C}_{6} \mathrm{D}_{6}$ in $\mathbf{4} \mathbf{h}$ in the mesophase region $\left(\mathrm{t}=70^{\circ} \mathrm{C}\right)$. The upper trace is of an aligned sample obtained by slowly cooling an isotropic solution within the magnetic field to the mesophase region. The bottom spectrum corresponds to a powder sample obtained by placing a disorder sample into the NMR probe at the mesophase temperature. 
sharp components is oberserved, indicates that the sample is ordered by the magnetic field during the isotropic $\rightarrow$ mesophase transition. From this spectrum alone it is however not possible to determine the orientation of the liquid crystalline domains with respect to the external field. To do so, we compared this result with that of a "powder" spectrum of the same sample and at the same temperature. Such a sample is obtained by allowing the isotropic solution to cool into the mesophase region outside the magnetic field and then introducing it into the NMR probe which has been preheated to the mesophase temperature region. The result is shown in the bottom trace of Fig. 5. The lineshape of this spectrum corresponds to an isotropic distribution of uniaxial domains. Since within the mesophase region there is no reorientation of the domains, it may be concluded that it is not nematic, but rather most probably columnar. Furthermore since the mesophase is uniaxial it is very likely that the columns are arranged in hexagonal arrays as in some of the conventional discotic phases.

Comparison of the two spectra in Fig. 5 indicates that the aligned sample (upper trace) consists of a two-dimensional distribution of domains whose directors all lie in a plane perpendicular to the field direction. This follows from the fact that the doublet splitting of the aligned samples matches the perpendicular features of the powder sample. The same result was also obtained for $\mathrm{C}_{6} \mathrm{D}_{6}$ dissolved in $\mathbf{4} \mathbf{d}$ and in 4f. We may thus conclude that the magnetic anisotropic susceptibility $\Delta \chi=\chi_{\perp}-\chi_{\perp}$ for these compounds and most probably for the whole series $(\mathbf{4} \mathbf{c}-\mathbf{k})$ is negative. A negative sign for $\Delta \chi$ was also found for triphenylene and truxene discotics although $\Delta \chi$ for the corresponding benzene hexaalkanoates series is apparently positive [7].

Similar spectra as those shown in the upper trace of Fig. 5 for $\mathbf{4 h}$ were observed for solutions of $\mathrm{C}_{6} \mathrm{D}_{6}$ in the mesophase region of the members $(\mathbf{4} \mathbf{c}-\mathbf{k})$ ( $n=7$ to 15 ) of the series 4. In Fig. 6 the temperature dependence of the quadrupole splittings, $v_{\mathrm{Q}}$, for the whole series is plotted. It may be seen that within the mesophase region in all cases there is a rather steep decrease in $v_{\mathrm{Q}}$ with increasing temperature. Similarly, large temperature dependencies of $v_{\mathrm{Q}}$ of deuterated solute molecules were observed in the columnar phases of the conventional discotic liquid crystals, an effect which was interpreted in terms of a fast dynamic equilibrium between two solvation sites
[7]. The behaviour observed in the hexasulfone series $\mathbf{4}$ may therefore be taken as supporting evidence for a columnar structure for the mesophases of this series as suggested above.

In the high temperature end of the mesophase region the deuterium doublet collapsed into a single peak due to the isotropic phase. However in the low temperature end of the mesophase a sudden discontinuity in the splitting was usually observed, with a sharp change in the slope of the $v_{\mathrm{Q}}$ vers. temperature curve (see Fig. 6). On further cooling, the doublet

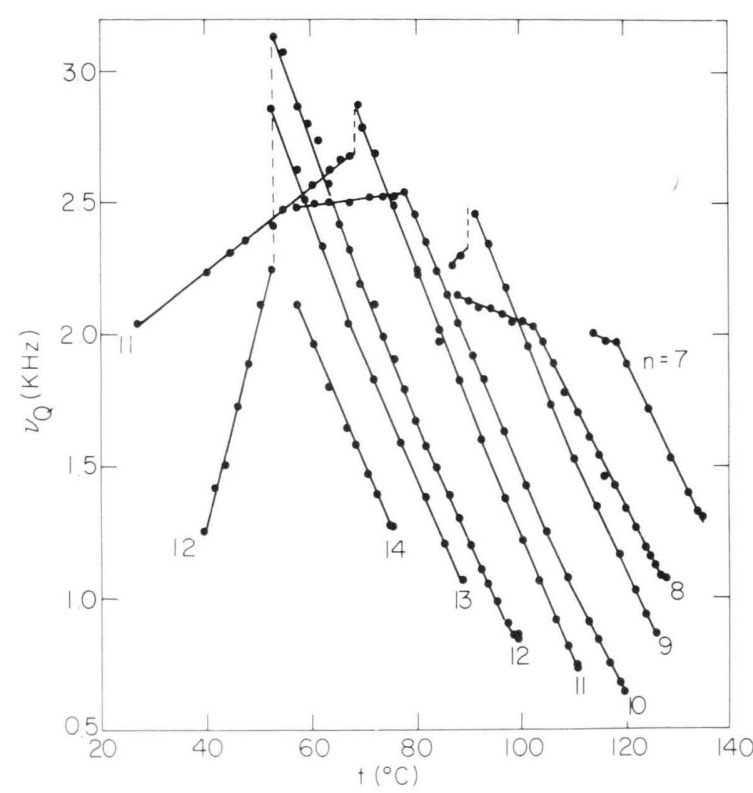

Fig. 6. The deuterium quadrupole splittings $v_{\mathrm{Q}}$ in hexakis(alkylsulfono)benzene solutions (of approximately 3 wt. $\left.\% \mathrm{C}_{6} \mathrm{D}_{6}\right)$ as function of temperature, $\mathbf{4} \mathbf{c}-\mathbf{j}, n=7-14$.

components usually broadened and finally disappeared. As an example we reproduced in Fig. 7 the spectra observed for $\mathrm{C}_{6} \mathrm{D}_{6}$ in $\mathbf{4} \mathbf{h}$. Note in particular the $98^{\circ} \mathrm{C}$ spectrum which consists of a superposition of isotropic and mesophase spectra, and the $52{ }^{\circ} \mathrm{C}$ trace which consists of a superposition of two mesophase spectra. The fact that "high resolution" spectra are observed in both regions may imply that below the normal mesophase other liquid crystalline mesophases occur. For the higher homologs, $\mathbf{4 i}$ and $\mathbf{4 j}$, this second mesophase was not observed and the spectrum disappeared due to solidification at the lower temperature end of the mesophase region. For $4 \mathbf{k}$ the mesophase containing the $\mathrm{C}_{6} \mathrm{D}_{6}$ solvent per- 


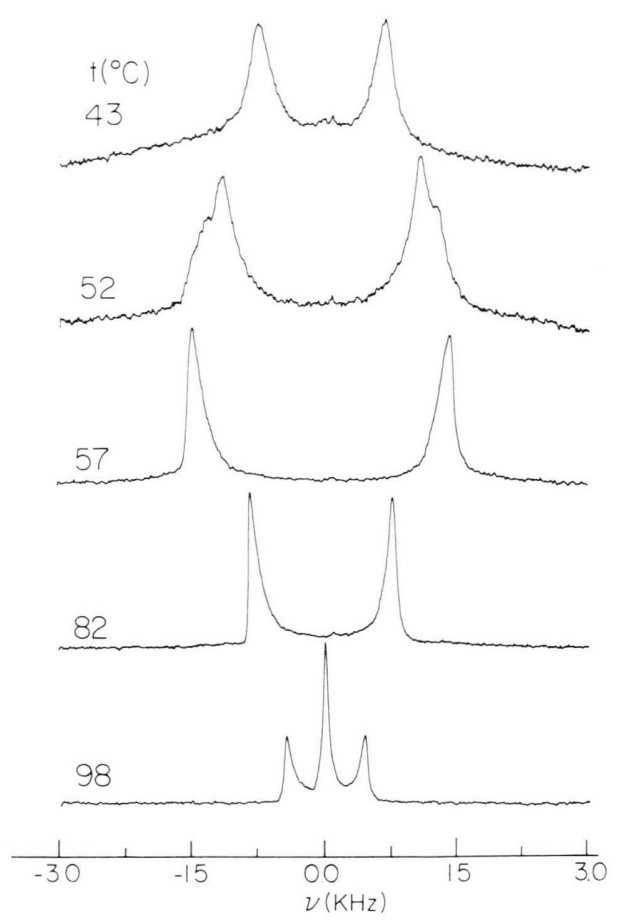

Fig. 7. Deuterium NMR spectra of a $2.9 \mathrm{wt} \%$ solution of $\mathrm{C}_{6} \mathrm{D}_{6}$ in $\mathbf{4 h}$ at different temperatures. Note the biphasic regions at $98^{\circ} \mathrm{C}$ and $52{ }^{\circ} \mathrm{C}$.

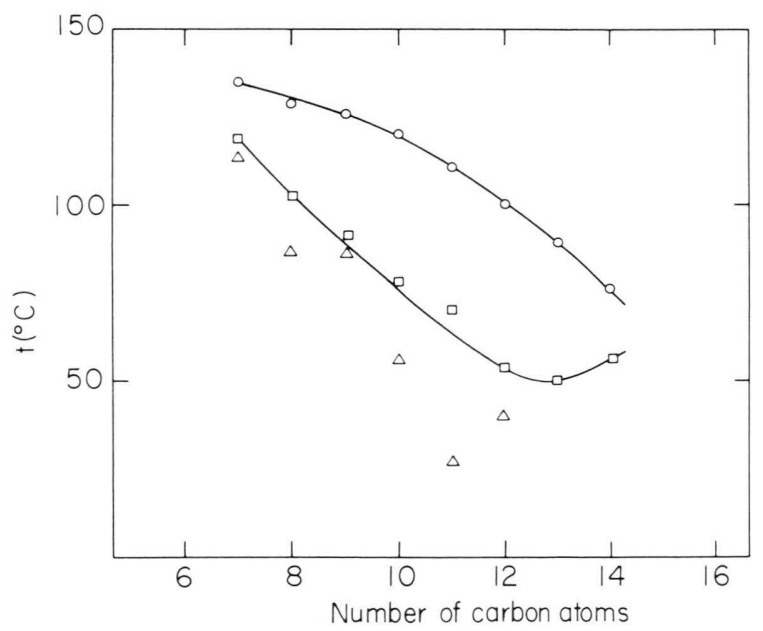

Fig. 8. Phase diagram of the hexasulfone series 4 based on the NMR results. The symbols are as in Fig. 2.

sisted for only a very narrow temperature range and the benzene spectrum consisted of a single broad line.

Using the curves of Fig. 6, a phase diagram based on the NMR results was constructed (see Fig. 8).
The overall results are quite similar to those obtained from the DSC and optical microscopy experiments, however the range of stability of the mesophase containing the benzene probe is somewhat narrower; there is a slight depression of the clearing point temperatures and a slight increase in the lower transition temperatures in particular for the higher homologs of the hexasulfone series 4 .

\section{Conclusions}

1. Hexakis(alkylsulfono)benzenes with chains containing between 7 and 15 carbons $(\mathbf{4} \mathbf{c}-\mathbf{k})$ exhibit discotic mesophases. The mesophases are uniaxial and most probably columnar with hexagonal symmetry. It appears that a minimum chain length is required for providing sufficient "lubrication" to facilitate molecular translation and reorientation in the mesophase, but the chains must also be sufficiently short in order not to slow down this motion too much.

2. The anisotropic magnetic susceptibility of the mesophases is negative and at sufficiently strong magnetic field they align perpendicular to the field direction.

3. There are several additional intermediates phases below the high temperature normal mesophase. It is not yet clear whether some of them are liquid crystals.

4. It appears from the temperature dependence of the solute NMR spectra that like in the conventional discotic liquid crystals the mesophases of the hexasulfones $4 \mathbf{c}-\mathbf{k}$ provide several distinct solvation sites for dissolved probe molecules.

This work was supported by the Deutsche Forschungsgemeinschaft, Bonn-Bad Godesberg, Germany, Project 116/8-1, E. Merck, Darmstadt, Germany, Fonds der Chemischen Industrie, Frankfurt/ Main, Germany, and by the Technical University Berlin (Partnershipprogram with the Weizmann Institute of Science, Rehovot, Israel, cooperation between Prof. Z. Luz and Prof. K. Praefcke). 
[1] Part XX of the series Liquid Crystalline Compounds; part XIX: B. Kohne, W. Poules, and K. Praefcke, Chemiker-Ztg. 108, 113 (1984). Also part LXIV of the series Organic Sulfurcompounds: part LXIII loc. cit.

[2] J. Billard, in Liquid Crystals of One and Two Dimensional Order, Ed. W. Helfrich and G. Heppke (1981), p. 383; C. Destrade, N. H. Tinh, and H. Gasparoux, Mol. Cryst. Liq. Cryst. 71, 111 (1981).

[3] D. Goldfarb, Z. Luz, and H. Zimmermann, Israel J. Chem. 23, 341 (1983).

[4] B. Kohne and K. Praefcke, Angew. Chem. 96, 70 (1984); Angew. Chem. Int. Ed. Engl. 23, 82 (1984).

[5] W. Poules and K. Praefcke, Chemiker-Ztg. 107, 310 (1983); 107, 374 (1983). - N. B.: In fact, prior to this preliminary communication another sulfur sontain- ing class of discotic liquid crystals had already been published: V. Gionis, R. Fugnitto, G. Meyer, H. Strzelecka, and J. C. Dubois, Mol. Cryst. Liq. Cryst. 90, 153 (1982); see further: H. Strzelecka, V. Gionis, J. Rivory, and S. Flandrois, J. de Phys. 44, C3-1201 (1983) as well as P. Davidson, A. M. Levelut, H. Strzelecka, and V. Gionis, J. de Phys. (Letters) 44, L823 (1983); K. Praefcke thanks to Dr. H. Strzelecka for this information from January 31, 1984.

[6] D. Demus and L. Richter, Texture of Liquid Crystals, Verlag Chemie, Weinheim 1978.

[7] D. Goldfarb, Z. Luz, and H. Zimmermann, J. Phys. 42, 1803 (1981); 43, 42 (1982); 43, 1255 (1982); D. Goldfarb, R. Poupko, Z. Luz, and H. Zimmermann, J. Chem. Phys. 79, 4035 (1983). 